${ }^{\odot}$ Entomologica Fennica. 31.VIII.1992

\title{
Neue und wenig bekannte Arten der Lepidopteren-Fauna Lettlands. 13. Mitteilung
}

\author{
Ivars Šulcs
}

Šulcs, I. 1992: Neue und wenig bekannte Arten der Lepidopteren-Fauna Lettlands. 13. Mitteilung. — Entomol. Fennica 3:99-104.

Faunistic and ecological notes are given on 66 Latvian species of Lepidoptera, 31 of which are new to the fauna. Of these, 13 are also new to Estonia and Lithuania: Ectoedemia albibimaculella (Larsen), E. longicaudella Klimesch, Tinea pallescentella Stainton, Bucculatrix noltei Petry, Agonopterix bipunctosa (Curtis), Coleophora conyzae Zeller, C. gardesanella Toll, Metzneria aestivella (Zeller), Scrobipalpa nitentella (Fuchs), Epinotia indecorana (Zetterstedt), Pammene clanculana (Tengström), Dichrorampha uralensis (Danilevsky), Eupithecia ochridata Pinker. 2341 species of Lepidoptera are now known from Latvia.

Ivars Šulcs, Stirnu iela 18, Riga-80, Latvia

Das Territorium Lettlands ist faunistisch nicht so gut erforscht wie die Nachbarländer Schweden, Finnland und Dänemark. Teilweise ist dies vermutlich mit der geringen Sammlerzahl in Lettland zu erklären, und auch die ökonomische Umstände in Lettland lassen nicht viel freie Zeit. In den Jahren 1988-1990 wurde in verschiedenen Ortschaften Lettlands gesammelt, die meiner Ansicht nach noch wenig erforscht waren. Als Resultat kann ich 31 Arten (mit * oder ** bezeichnet) als neu für Lettland melden, von welchen 13 neu für das ganze Balticum sind (mit ** bezeichnet). Für 25 andere Arten werden neue Angaben über die Verbreitung und Ökologie angeführt.

Am 1. Januar 1991 betrug die Gesamtzahl von Lepidoptera-Arten in Lettland 2341.

\section{Nepticulidae}

*Stigmella nanivora (W. Petersen, 1930)

Aus auf Torfmooren bei Mazsalaca an Betula nana gefundenen Minen mehrfach aufgezogen.
Die Frage, ob $S$. nanivora mit $S$. betulicola (Stainton, 1865) identisch ist, bleibt noch offen.

*Stigmella oxyacanthella (Stainton, 1854)

Rundāle, 1990 mehrere von Crataegus gesammelte Exemplare aufgezogen

*Stigmella myrtillella (Stainton, 1857)

Kemeri, 1990-08-05 ein $\sigma^{\top}$ am Licht in Mischwald.

*Stigmella pretiosa (Heinemann, 1862)

Priekuli, 1989-06-09 und Liepna, 1990-0624, je ein Exemplar auf Wiesen mit üppiger Vegetation aufgescheucht.

*Stigmella poterii (Stainton, 1857)

Mazsalaca, 1988-06-05 auf Torfmoor ein o', leg. G. Zēbergs. 
*Trifurcula headlayella (Stainton, 1854)

Grobina, 1990-07-04 mehrere Exemplare auf Wiesen am Liepāja-See (Leg. S. Kerppola, J. Junnilainen und I. Šulcs).

**Ectoedemia albibimaculella (Larsen, 1927)

Garkalne, 1990-07-10 mehrere Exemplare von Arctostaphylus aufgescheucht.

**Ectoedemia longicaudella Klimesch, 1953

Virga (SW-Lettland), ein ơ 1988-07-26 am Licht in einem Eichenhain.

\section{Ectoedemia klimeschi (Skala, 1933)}

An Stämmen von Populus alba in Riga, nicht häufig.

\section{Adelidae}

\section{Adela leucocerella (Scopoli, 1763)}

Die Art war für Lettland schon von F. Lienig gemeldet. Im Juni 1990 war diese Art im Daugava-Tal bei Riteri an Blüten von Veronica teucrium häufig

\section{Cauchas rufimitrella (Scopoli, 1763)}

Virga, 1990-05-27 von verschiedenen Brassicaceae (Raphanus u. a.) mehr als 15 Exemplare mit dem Kescher gefangen. Ebenso auch bei Liepna (NO-Lettland) am 1990-06-24 ein Exemplar.

\section{Tischeridae}

Tischeria heinemanni Wocke, 1871

Anscheinend kommt diese Art in Lettland nur auf Torfmooren vor. So fing ich bei Mazsalaca sehr nahe der estnischen Grenze am 1988-06-05 zwei Exemplare. Vorher nur im vorigen Jahrhundert von C. A. Teich bei Salaspils ebenfalls auf Torfmoor gefunden.

\section{Tineidae}

Archimeesia irinae Savenkov, 1989

Diese aus Lettland beschriebene Art ist hier bisher nur an zwei Stellen beobachtet worden zwei Exemplare im Naturschutzgebiet Slitere und ein drittes Stück in einem alten Park im südlichen Lettland (Saulite). Ich fing ein Mänchen bei sehr günstigen Lichtverhältnissen am Licht in Slitere am 1987-07-21 am alten Ufer der Ostsee, das mit primärem Wald bedeckt ist.

\section{**Tinea pallescentella Stainton, 1851}

Mehrere Exemplare in Riga in Räumen der Rigaer Technischen Universität im Frühjahr 1990 gefunden.

\section{Tinea steueri G. Petersen, 1966}

Priekuli, 1989-06-03 ein $९$ am Licht in einem Walddickicht gefangen. Zweites Exemplar für Lettland.

\section{Gracillariidae}

Ornixola caudulatella (Zeller, 1939)

Es war eine grosser Überraschung für mich, von dieser mehr im östlichen Europa gefundenen Art in SW-Lettland (Virga) am 1988-06-08 in einer Buschwiese ein frisches Exemplar am Licht $\mathrm{zu}$ fangen. Im vorigen Jahrhundert in Lettland auch von F. Lienig bei Koknese und von C. A. Teich in Dubulti gefangen.

*Phyllonorycter anderidae (W. Fletscher, 1885)

Im Jahre 1989 aufgezogen aus Betula nanaMinen, die auf einem Torfmoor bei Mazsalaca nahe der estnischen Grenze gefunden wurden.

\section{Bucculatricidae}

**Bucculatrix noltei Petry, 1902

Liepna, 1990-06-09 ein o an trockener Stelle fliegend gefangen. Die nächsten Fundorten in Richtung Süden liegen im nördlichen Polen. 


\section{Lyonetiidae}

*Leucoptera lustratella (Herrich-Schäffer, 1855)

In Vilzēni (nördliches Lettland) im Jahre 1989 zusammen mit Minen von Ectoedemia septembrella eingetragen und aufgezogen. In Jumprava an einer Flussterrasse der Daugava 199006-27 mehrere Exemplare von Hypericum perforatum aufgescheucht.

\section{Oecophoridae}

Agonopterix parilella (Treitschke, 1835)

Von Peucedanum oreoselinum im Jahre 1990 aufgezogen und auch als Imago am Licht an mehreren Stellen längs des Flusses Daugava, so etwa in Jaunogre, Jumprava und Riteri.

\section{**Agonopterix bipunctosa (Curtis, 1850)}

Diese nur von England und Schweden gemeldete Art kommt in SW-Lettland vor — am 1989-07-30 kam ein $0^{7}$ auf Wiesen des Liepāja-Sees, aus Licht auf denen auch die Futterpflanze der Raupe, Serratula tinctoria, in grossen Mengen vorkommt.

\section{Elachistidae}

\section{Elachista zernyi Hartig, 1941}

Diese bisher nur aus dem Süden Europas gemeldete und von E. Traugott-Olsen determinierte Art wurde an drei Stellen in Lettland an trockenen Kiefernwaldschonungen mit Heidekraut festgestellt: Dreilini bei Riga, Daugavpils und Tērvete. Die Raupe fanden wir Mitte Mai an Koeleria sp. und haben auch Falter aufgezogen. Als Falter im Freien in Dreilini 1985-06-10 drei Exemplare, in Tērvete 1986-06-03 ein Exemplar, in Daugavpils 1987-05-29 zwei Exemplare.

\section{Coleophoridae}

*Coleophora gryphipennella (Hübner, 1796)

In Riga, in der Umgebung Rigas die Säcke im Herbst an Rosa, auch in Kemeri am Licht 1989-06-16 zwei Exemplare gefangen.
Coleophora ptarmicia Walsingham, 1910

Die Verbreitung dieser Art ist in Lettland nur auf den südostlichen Teil beschränkt. Ausser am früheren Fundort Škaune fand ich auch bei Auleja am 1988-06-29 zwei Säcke an Achillea millefolium aus welchen am 1988-06-20 und 198806-25 Falter schlüpften.

**Coleophora conyzae Zeller, 1868

Es existiert eine spärliche Population im grossen Kemmernschen Waldmassiv ca $40 \mathrm{~km}$ westlich von Riga. Ich fing 1988-06-29 und 198906-16 je ein Exemplar.

\section{Coleophora scabrida Toll, 1959}

An sandigen Stellen bei Daugavpils am 199006-01 von Herniaria zwei Exemplare aufgescheucht. Im Juli 1990 drei Säcke an Herniaria bei Bārta in SW-Lettland.

\section{**Coleophora gardesanella Toll, 1959}

Ein Exempar dieser Seltenheit, ein Weibchen, wurde am 1990-07-21 an einer Kiesgrube mit Bewuchs von Artemisia campestris, Anthemis tinctoria, Centaurea scabiosa und Centaurea jacea in NO-Lettland bei Liepna gegen Abend gefangen. Die Genitalstruktur stimmt gut mit der Abbildung von G. Baldizzone 1981 überein.

\section{Coleophora carelica Hackman, 1945}

Diese Art hat eine nördliche Verbreitung in Lettland. Die Säcke kann man im Juni lokal nicht selten an Kiefernwaldrändern und Kiesgruben an Achillea millefolium zu finden. Ich fand im Jahre 1990 bei Saule sogar an Artemisia vulgaris Säcke, aus denen Mitte Juli und Anfang August mehrere Exemplare schlüpften.

\section{Scythrididae}

*Scythris cicadella (Zeller, 1839)

Auf dürren, sandigen Flächen bei Daugavpils am 1990-06-28 zwei Exemplare mit dem Kescher gefangen. 
Scythris palustris (Zeller, 1855)

Die Art kommt vereinzelt in Lettland sowohl auf sumpfigen Wiesen als auch auf echten Torfmooren vor. So fing ich in Mazsalaca nahe der estländischen Grenze am 1989-07-06 auf einem Torfmoor ein Stück.

\section{Gelechiidae}

**Metzneria aestivella (Zeller, 1839)

Auf einer mit viel Carlina bewachsenen kalkhaltigen Stelle bei Kandava am 1988-06-24 ein frisches Exemplar.

Aristotelia caeruleopictella (Caradja, 1920) ssp. baltica A., I. Šulcs, 1983

Am 1990-07-27 fing ich bei Riteri am Ufer der Daugava (zweiter Fundort in Lettland) an einer kalkhaltigen, mit einzelnen Rhamnus frangula und Juniperus-Sträuchern bewachsenen Stelle ein frisches Exemplar. Im Unterwuchs fand sich viel Inula salicina - ganz ebenso wie am ersten Fundort bei Kandava. Die Raupe lebt in Litauen in zusammengesponnenen Blättern von Rhamnus frangula (P. Ivinskis, pers. comm.).

\section{Monochroa divisella (Douglas, 1850)}

Diese nur für England und Dänemark verzeichnete Art wurde schon an drei Stellen Lettlands gefunden - Carnikava, 1980-07-13, Jumprava, 1989-07-09 und Kemeri, 1989-07-27 je in einem Exemplar am Licht gefangen. Ein typisches Biotop — feuchte Buschheuschlage an Flussufern.

\section{Scrobipalpa klimeschi Povolny, 1967}

Zerstreut über Lettland verbreitet, jedoch im östlichen Teil häufiges. Ich fand diese Art in Šķaune, 1979-06-03 ein o, in Atašiene, 1988-0528 ein o, und in Liepna, 1990-06-09 ein o..

\section{*Scrobipalpa proclivella (Fuchs, 1886)}

In Naujene (SO-Lettland) an einer trockenen Anhöhe am 1990-06-02 ein Exemplar, ein zweites Exemplar am 1990-06-17 von Artemisia absinthium gesammelt und aufgezogen.
**Scrobipalpa nitentella (Fuchs, 1902)

Kuiviži, am 1983-06-27 ein Exemplar am Meeresstrand.

\section{Tortricidae}

Cnephasia alticolana (Herrich-Schäffer, 1851)

Im Gegensatz zur ähnlichen C. stephensiana fliegt diese Art bedeutend früher und man kann auf diese Weise frische Stücke dieser beiden Arten leicht vorläufig von einander unterscheiden. In Einzelnstücken an mehreren Orten in Lettland entdeckt. Am 1990-05-23 fing ich ein frisches Stück in einem Mischwald bei Ropaži.

\section{Eupoecilia sanguisorbana (H-S., 1851)}

Auf Wiesen am Liepāja-See bei Grobina in SWLettland nicht häufig gegen Abend fliegend und auch am Licht am 1989-07-30 und 1990-07-04.

**Epinotia indecorana (Zetterstedt, 1839)

Auf Torfmoor bei Mazsalaca am 1988-09-21 ein Exemplar von Betula nana aufgescheucht.

**Pammene clanculana (Tengström, 1869)

Im nördlichen Lettland (Mazsalaca) sehr nahe der estnischen Grenze auf Torfmoor von Betula nana am 1988-06-05 mehrere Exemplare aufgescheucht.

*Dichrorampha cinerascens (Danilevsky, 1948)

An trockenen Kiesstellen in NO-Lettland bei Liepna am 1989-07-14 und 1990-07-22 eine Anzahl von Exemplaren gefangen. In Nordeuropa war diese Art auch schon von Finnland und Estland bekannt.

**Dichrorampha uralensis (Danilevsky, 1948)

Diese Art wurde am 1990-06-01 an nassen, teilweise überschwemmten Wiesen der Daugava bei Daugavpils in mehreren Exemplaren entdeckt. An der Fundstelle wuchsen reichliche Bestände von Achillea ptarmica ssp. cartillaginea, der vermutlichen Futterpflanze dieser Art. Bisher war diese Art nur vom östlichen Russland bekannt. 


\section{Sesiidae}

Bembecia triannuliformis (Freyer, 1842)

Schon F. Lienig hat diese Art für Lettland angeführt. Bei Liepna (NO-Lettland) am 199006-24 ein Exemplar in einer Kiesgrube von Rumex acetosa aufgescheucht. Wurde auch von östlichen Teilen Estlands und Litauens gemeldet.

\section{Pterophoridae}

* Capperia trichodactyla (Denis \& Schiffermüller, 1775)

Im Juni 1990 wurde diese Art an vier Stellen (Riga, Riteri, Daugavpils, Naujene) als Raupe an Leonurus cardiaca gefunden. Am 1990-06-25 schlüpfte ein bei Daugavpils gesammeltes Exemplar aus.

\section{Pyralidae}

*Achroia grisella (Fabricius, 1794)

Am 1989-08-15 fing sich bei Jumprava in unberührter natürlicher Umgebung ein Exemplar in Selbstfänger. Verschiedene mündliche Mitteilungen über das Vorkommen dieser Art in Lettland waren bekannt, jedoch nicht mit Belegstiicken bestätigt gewesen.

\section{Hesperiidae}

Pyrgus serratulae (Rambur, 1839)

In Lettland sehr lokale und seltene Art, die bisher bei Riga und im äussersten Osten in Šḳaune gefunden worden war. Am 1988-06-01 und 198806-03 fand ich in Garkalne am Abend an trockenen Artemisia campestris -Stengeln sitzend zwei frische Exemplare.

\section{Lycaenidae}

*Maculinea teleius (Bergsträsser, 1779)

Bei Grobina (SW-Lettland), fing ich in Gegenwart der finnischen Entomologen S. Kerppo- la und J. Junnilainen am 1990-07-04 auf Wiesen am Liepāja-See ein nicht ganz frisches Exemplar. In diesem Biotop wächst in ausgedehnten Beständen die Futterpflanze dieser Art Sanguisorba officinalis. Die nächsten Fundorte liegen im südlichen Litauen.

\section{Geometridae}

*Eupithecia cauchiata (Duponchel, 1830)

Hierher gehört ein genitalisch überprüftes Weibchen, welches sich am 1989-06-22 bei Kemeri in einem Buschheuschlag in meinem Selbstfänger fing.

\section{**Eupithecia ochridata Pinker, 1968}

In unserer Sammlung stehen mehrere Exemplare von Riga und Silene, welche schon in den dreissiger Jahren dieses Jahrhunderts gefangen worden sind.

\section{Alcis maculatus (Staudinger, 1892)}

Bisher nur aus dem nordöstlichen Teil Lettlands bekannt. So fand ich von dieser Art bei Liepna am 1989-07-22 und 1990-07-21 je ein frisches Exemplar, am 1990-08-17 zwei abgeflogene Exemplare. Weiter östlich ist diese Art auch bei Luga und im Gebiet um St. Petersburg festgestellt worden.

\section{Arctiidae}

*Pelosia obtusa (Herrich-Schäffer, 1852)

Diese aus Litauen und Südschweden gemeldete Art erwarten wir auch in Lettland. Bei günstigen Lichtverhältnissen kam am 1989-0630 in Virga ein frisches Exemplar aus Licht.

\section{Noctuidae}

*Schrankia taenialis (Hübner, 1809)

Am 1990-07-05 in Carpinus-Wald wurde in SW-Lettland (Lukna) vom finnischen Entomo- 
logen J. Junnilainen ein ganz frisches Stück aufgescheucht.

\section{Xylomoia strix Mikkola, 1980}

Diese Relikt-Art kommt regelmässig im Gauja-Flusstal bei Turaida vor. So fing ich am 1988-06-25 bei sehr günstigen Lichtverhältnissen zwei ơ und drei $0^{7} \sigma^{7}$ am Licht.

\section{Oligia fasciuncula (Haworth, 1809)}

Bisher nur von SW-Lettland bekannt. Am 1989-06-30 kamen in Virga zwei nicht ganz frische Exemplare ans Licht.

\section{Hydraecia petasitis Doubleday, 1848}

Das für Lettland zweite Exemplar kam am 1989-08-21 bei Skriveri am Rande eine grossen Petasites officinalis-Bestandes ans Licht.

\section{Heliophobus kitti Schawerda, 1917}

Ausser an dem Fundort in unserem Naturschutzgebiet Šķaune im äussersten Osten Lettlands, wo wir diese Art in den siebziger Jahren konstatierten, kam am 1990-06-28 auch in der Umbegung von Daugavpils am Rande eines sandigen Kiefernwaldes, der an reich mit Helichrysum, Artemisia campestris, Silene otitae, Herniaria, Potintilla sp. u.a. bewachsenes steppenartigen Dünenland angrenzte, ein Pärchen ans Licht.

\section{Literatur}

Baldizzone, G. 1981: Contributions á la connaissance des Coleophoridae XXII. — Nota Lepidopterol. 4(3):6379.

Danilevskii, A. S., Kuznetsov V. I. (Данилевский, А. С., Кузнецов В. И.) 1968: [Insects, Lepidotera V. 1. Tortricidae, Lasperyesiini.] (In Russian) — Fauna USSR, N.S. 98.636 pp.
Heath, J. 1976: The moths and butterflies of Great Britain and Ireland. - Curwen Press. 343 pp.

Ivinskis, P. 1982: Lepidoptera species new to the Lithuanian SSR, found in 1968-1982. (In Russian with English summary) - In: Ionaitis, V. (ed.), New and rare for the Lithuanian SSR insect species: 28-47. Akad. Nauk Litovskoj SSR, Vilnius. 81 pp.

Kerppola, S. 1981: Dichrorampha conerascens (Lepidoptera, Tortricidae) in Finland. - Notulae Entomol. 61:157-158.

Kaila, L. 1989: Eupithecia ochridata Pinker new to northern Europe: morphological and biological studies on the E. innotata complex (Lepidoptera, Geometridae). — Notulae Entomol. 69:39-45

Nolcken, J. H. W. 1870: Lepidopterologische Fauna von Estland, Livland und Kurland. - Arbeiten Naturforscher-Ver. Riga. Riga. 849 pp.

Palm, E. 1989: Nordeuropas prydvinger (Lepidoptera, Oecophoridae). — Fauna Bøger, København. 247 pp.

Sattler, K. 1986: Die an Compositen gebundenen Scrobipalpa-Arten des östlichen Österreichs (Lepidoptera, Gelechiidae). - Ann. Naturhist. Mus. Wien 88/ 89:435-456.

Savenkov, N. 1989: A new species of genus Archimeesia Zag. (Lepidoptera, Tineidae) from Latvia. (In Russian with English summary) - Latvijas Entomol. 32:9294.

- 1990: New data about the Lepidotera fauna of Latvia. (In Russian with English summary) - Latvijas Entomol. 33:90-95.

Svensson, I. 1971: Scandinavian Bucculatrix Z. (Lep. Bucculatricidae). — Entomol. Scand. 2:99-109.

Šulcs, A. 1978: Heliophobus texturata ssp. kitti, eine für Lettland neue Noktuide (Lepidoptera, Noctuidae). Notulae Entomol. 58:27-31.

Šulcs, A., Viidalepp, J. \& Ivinskis, P. 1981: Nachtrag zur Verbreitung der Grosschmetterlinge im Baltikum. Deutsche Entomol. Zeitung, N.F. 28(I-III):123-146.

Šulcs, A. \& Šulcs, I. 1983: Neue und wenig bekannte Arten der Lepidopteren-Fauna Lettlands. 9. Mitteilung. Notulae Entomol. 63:37-48.

- 1984: Neue und wenig bekannte Arten der LepidopterenFauna Lettlands. 10. Mitteilung. — Notulae Entomol. 64:151-155.

Traugott-Olsen, E. \& Schmidt Nielsen, E. 1977: The Elachistidae (Lepidoptera) of Fennoscandia and Denmark. - Fauna Entomol. Scandinavica 6. Scandinavian Science Press Ltd., Klampenborg, Denmark. 299 pp.

Received 16.IV.1991 